\title{
Modelos de determinação não-destrutiva da área foliar em girassol
}

\author{
Models for estimating leaf area in sunflower
}

\author{
Ivan Carlos Maldaner ${ }^{\mathrm{I}}$ Arno Bernardo Heldwein ${ }^{\mathrm{II}}$ Luis Henrique Loose ${ }^{\mathrm{III}}$ \\ Dionéia Daiane Pitol Lucas ${ }^{\mathrm{III}}$ Fabrício Ivan Guse ${ }^{\mathrm{I}}$ Mateus Possebon Bortoluzzi"II
}

\section{RESUMO}

Os objetivos deste trabalho foram obter e testar modelos matemáticos de estimativa da área do limbo foliar em função das suas dimensões lineares para o girassol. Foram conduzidos dois experimentos na área experimental do departamento de Fitotecnia da Universidade Federal de Santa Maria. As plantas de girassol foram coletadas a partir dos 27 dias após emergência (DAE). A área foliar (AF) foi determinada pelo método dos discos. Ajustaram-se modelos lineares, quadráticos, cúbicos e potenciais entre área foliar e comprimento ou largura e seus produtos (comprimento*largura), sendo eliminados os que apresentaram coeficiente de determinação menor do que 0,90. A estatística utilizada para avaliar o desempenho dos modelos foi a raiz do quadrado médio do erro (RQME). Os modelos que melhor se ajustaram aos dados foram: potência, quadrático e cúbico, considerando a largura como variável independente. A área foliar de girassol pode ser estimada com o modelo potência, por ser o mais preciso, e a largura da folha.

Palavras-chave: Helianthus annuus, modelagem, dimensão foliar.

\section{ABSTRACT}

The objective of this study was to obtain and to numerical models to estimate the leaf area in function leaves linear dimension in sunflower. Two experiments were conducted at the experimental area of the Plant Science Department of the Federal University of Santa Maria, Santa Maria, RS, Brazil. Plants of sunflower were collected starting 27 days after emergency (DAE). The disks method was used to determine the leaf area (LA). Leaves were dried in oven at $65^{\circ} \mathrm{C}$ until constant weight. Linear, quadratic, cubic and power models between leaf area and length or width, and the product (length * width), were fitted. Models that apresented coefficient of determination lower than 0.90 were not selected. The statistic used to evaluate the performance of the models was the root mean square error (RQME). Models that had the best fit were power, quadratic and cubic using blade width as the independent variable. Leaf area in sunflower can be estimated with the power model, which was the most accurate, with width of the leaf.

Key words: Helianthus annuus, modeling, leaf dimensions.

\section{INTRODUÇÃO}

A área foliar (AF) de uma cultura interfere na interceptação da radiação solar e na troca de água e energia entre a folha e o ar adjacente à atmosfera. A folha é o principal órgão das plantas envolvido na fotossíntese e responsável pelas trocas gasosas entre a planta e o ambiente (PEREIRA et al., 1997). O processo fotossintético depende da interceptação da radiação solar e sua conversão em energia química. Portanto, o índice de área foliar (IAF) pode ser considerado um dos parâmetros indicativos de produtividade (FAVARIN et al., 2002). Por isso, medições precisas de AF são essenciais para entender a interação entre o crescimento da planta e o ambiente (DE JESUS et al., 2001). O IAF é necessário para estudos agronômicos e fisiológicos envolvendo crescimento vegetal (BLANCO \& FOLEGATTI, 2003) e útil na avaliação de

IPrograma de Pós-graduação em Engenharia Agrícola, Universidade Federal de Santa Maria (UFSM), Santa Maria, RS, Brasil.

IIDepartamento de Fitotecnia, Centro de Ciências Rurais (CCR), UFSM, 97105-900, Santa Maria, RS, Brasil. E-mail: heldwein@smail.ufsm.br. Autor para correspondência.

${ }^{\text {IIIC }}$ urso de Agronomia, CCR, UFSM, Santa Maria, RS, Brasil. 
várias práticas culturais, tais como densidade de plantio, adubação, irrigação, poda e aplicação de defensivos (FAVARIN et al., 2002).

A área foliar total da planta pode ser obtida por métodos diretos ou indiretos. Os métodos diretos são métodos destrutivos e exigem a retirada da folha ou de outras estruturas, o que muitas vezes não é possível devido ao limitado número de plantas na parcela experimental. Os métodos indiretos são nãodestrutivos e podem fornecer estimativas precisas da AF desde o início até o fim do ciclo acompanhando-se a mesma planta (PEKSEN, 2007). Os mais utilizados são os aparelhos integradores de área foliar e os modelos matemáticos com dimensões lineares como variáveis de entrada no modelo. Os aparelhos integradores de área foliar são práticos para medir a AF, porém seu uso é pouco difundido devido ao alto custo para sua aquisição. Os modelos matemáticos envolvem medições lineares, tais como comprimento foliar e largura foliar, ou alguma combinação dessas variáveis, geralmente, têm boa precisão para estimar a AF real (BLANCO \& FOLEGATTI, 2005). São acessíveis em termos de custo, pois é preciso apenas uma régua graduada para se medirem as dimensões foliares. COELHO FILHO et al. (2005) avaliaram a correlação entre variáveis biométricas e área foliar em plantas de lima ácida "taipe” e indicaram a utilização de diâmetro de tronco e ramos para estimar AF de forma rápida, simples, não-destrutiva e aplicável a campo.

Os métodos de determinação da AF nãodestrutivos são elaborados a partir da função do produto entre comprimento e largura do limbo foliar, como feito para o meloeiro (NASCIMENTO et al., 2002), feijão-vagem(QUEIROGA et al., 2003), pepineiro (NIED et al., 2001), berinjela (COSTA et al., 2007), girassol (ROUPHAEL et al., 2007), dentre outras culturas, porém estes modelos nem sempre são estatisticamente melhores do que aqueles gerados com apenas uma das dimensões do limbo foliar (BLANCO \& FOLEGATTI, 2000; NASCIMENTO et al., 2002; QUEIROGA et al., 2003; COSTA et al., 2007; ROUPHAEL et al., 2007).

Os objetivos do presente trabalho foram obter e testar modelos matemáticos de estimativa da área do limbo foliar em função das suas dimensões lineares para o girassol.

\section{MATERIAL E MÉTODOS}

Foram realizados dois experimentos conduzidos na primavera de 2007 e no verão/outono de 2008, utilizando-se os genótipos Aguará 03 e Hélio 358 (latitude: 2943’23”S, longitude: 5343’15’W e altitude: $95 \mathrm{~m})$.

O clima da região, segundo a classificação de Köppen, é do tipo Cfa, subtropical úmido com verões quentes (MORENO, 1961). O solo foi classificado como “Argissolo Vermelho distrófico arênico” (EMBRAPA, 1999), apresentando um horizonte superficial Ap, com transição para horizonte B textural acerca de 0,3m de profundidade. A correção do solo foi feita com base na sua análise química. A adubação utilizada na semeadura foi de $80 \mathrm{~kg} \mathrm{ha}^{-1}$ de Bórax, $18 \mathrm{~kg} \mathrm{ha}^{-1}$ de N, $44 \mathrm{~kg} \mathrm{ha}^{-1}$ de $\mathrm{P}_{2} \mathrm{O}_{5}$ e $41 \mathrm{~kg} \mathrm{ha}^{-1}$ de $\mathrm{K}_{2} \mathrm{O}$. Em cobertura, foram aplicados $42 \mathrm{~kg} \mathrm{ha}^{-1}$ de N 30 dias após a emergência das plantas.

O espaçamento foi de $0,90 \mathrm{~m}$ entre fileiras de plantas e $0,25 \mathrm{~m}$ entre plantas na fileira, obtendo-se uma população final de 44.440 plantas por hectare. As semeaduras foram realizadas na profundidade de 3 a $4 \mathrm{~cm}$, em uma área de $1250 \mathrm{~m}^{2}$, nas datas de 04/09/2007 e 21/01/2008, ocorrendo a emergência nas datas de 12/ 09/2007 e de 30/01/2008. No experimento da primavera de 2007, foram coletadas 10 amostras de plantas do genótipo Hélio 358 e Aguará 03 ao longo do ciclo da cultura. Foram amostradas três plantas aos 27 e aos 34 dias após a emergência (DAE). As demais amostras foram coletadas aos 45, 60, 74 e $81 \mathrm{DAE}$, permitindo, assim, abranger todos os tamanhos das folhas.

No experimento de verão/outono de 2008, foram coletadas sete amostras de plantas de cada genótipo ao longo do ciclo da cultura, sendo a primeira coleta em 26/02/2008 e a última em 05/04/2008. Foram amostradas duas plantas de cada genótipo aos 27 e 35 DAE. As demais amostragens foram feitas aos 49, 59 e 66 DAE, coletando-se uma planta de cada. Os modelos foram gerados com dados da primavera de 2007 e testados com os dados de 2008.

As folhas fotossinteticamente ativas e nãodanificadas foram separadas das demais partes da planta na inserção entre o limbo e o pecíolo. Em cada folha, mediram-se comprimento (C) ao longo da nervura central, considerando-se a distância desde o ápice da folha até a inserção do limbo com o pecíolo, e a maior largura (L) perpendicular ao alinhamento da nervura central, ambos em cm, utilizando-se uma régua graduada em milímetros. Com auxílio de um calador com diâmetro de 30mm, obteve-se o número máximo possível de discos do limbo, incluindo as nervuras.

As amostras dos discos e o restante da folha foram acondicionados separadamente em sacos de papel e levados à estufa de secagem, com aeração 
forçada e temperatura de $65^{\circ} \mathrm{C}$, até peso constante. A massa de matéria seca das duas partes foi determinada com auxílio de uma balança eletrônica com resolução de $1 \mathrm{mg}$ e precisão de $5 \mathrm{mg}$. A área total de cada folha (AF), em $\mathrm{cm}^{2}$, foi calculada por: AF $=$ MST * ATD * $\mathrm{MSD}^{-1}(1)$, em que "MSD” é a massa seca dos discos, “ATD” área total dos discos e "MST" a massa seca total das folhas.

Uma vez conhecida a área do limbo da folha, foi determinada sua relação com as dimensões lineares (C ou L) e seu produto (C L), por meio do ajuste de modelos matemáticos aos dados. Foram obtidos modelos lineares, quadráticos, cúbicos e potenciais. Obteve-se também modelo de regressão linear, quadrática e cúbica com passagem forçada da função pela origem, tendo em vista que essa forma de modelo é mais apropriada para estimar a área foliar (LAKITAN, 1989), pois apresenta bases geométricas aceitáveis. A escolha dos melhores modelos foi feita entre os que apresentaram valores de coeficiente de determinação $\left(\mathrm{R}^{2}\right)$ maiores que 0,90. Para a avaliação do desempenho dos modelos, a estatística utilizada foi a raiz quadrada do quadrado médio do erro (RQME) calculada por (JANSSEN \& HEUBERGER, 1995):

$$
\mathrm{RQME}=\left(\Sigma\left(\mathrm{s}_{\mathrm{i}}-\mathrm{o}_{\mathrm{i}}\right)^{2} / \mathrm{N}\right)^{0,5}
$$

em que "s” é o valor estimado, “o” é o valor observado e N é o número de observações. Para a geração dos modelos, o N é = a 202 folhas e para o teste o N é =169 folhas. Quanto menor o RQME, melhor o modelo.

Também foi testado o modelo AF $=6,72+$ $0,65(\mathrm{~L})^{2}\left(\mathrm{R}^{2}=0,978\right.$ e $\left.\mathrm{QME}=985\right)$, obtido por ROUPHAEL et al. (2007), na Itália, para verificar se esse modelo poderia ter validade para os genótipos de girassol cultivados nas condições do Brasil.O modelo obtido na Itália foi testado com um conjunto de 371 folhas obtidas de dois genótipos de girassol (Hélio 358 e Aguará 03). A estatística usada para a avaliação do modelo foi o RQME, e o valor de p do modelo foi menor que $0,0001(\mathrm{P}<0,0001)$. A geração e avaliação dos modelos foram feitas no software SAS system for Windows 9.0.

\section{RESULTADOS E DISCUSSÃO}

Foram obtidos 10 modelos estimadores da área foliar que apresentaram coeficientes de determinação elevados $\left(\mathrm{R}^{2}>0,90\right)$. Seu teste é apresentado na tabela 1 . Todos os modelos apresentaram teste significativo $(\mathrm{P}<0,0001)$ para estimar a AF, em função do C e/ou L. Os modelos potência, quadrático e cúbico apresentaram valores de coeficientes de determinação $\mathrm{R}^{2}$ superiores aos modelos lineares. O melhor resultado foi obtido com o modelo não-linear de potência, utilizando-se apenas a largura (L), pois apresentou maior $\mathrm{R}^{2}$ na geração do modelo e melhor desempenho no teste, com um menor valor de RQME em relação aos modelos quadráticos e cúbicos. O modelo não-linear potência utilizando a largura é mais realístico para estimar a área foliar do girassol em folhas menores, pois, para uma folha com $1 \mathrm{~cm}$ de largura, esse modelo estima uma $\mathrm{AF}$ de $1,75 \mathrm{~cm}^{2}$, já, no modelo quadrático, a AF seria de $4,1 \mathrm{~cm}^{2}$ e, no cúbico, 3,9 $\mathrm{cm}^{2}$. Assim, o modelo de potência é o mais indicado por apresentar um RQME menor e um coeficiente angular do teste mais próximo de um, em relação aos demais modelos (Figura 1).

Tabela 1 - Modelos de regressão para estimativa da área foliar (AF, $\mathrm{cm}^{2}$ ) de girassol e respectivos coeficientes de determinação de geração do modelo $\left(\mathrm{R}^{2}\right)$, coeficiente angular do teste do modelo (a), coeficiente linear do teste do modelo, coeficiente de determinação do teste do modelo $\left(\mathrm{R}^{2}\right)$ e raiz quadrada do quadrado médio do erro (RQME), em função do comprimento (C) e da largura máxima (L) das folhas e do seu produto $\left(\mathrm{C}^{*} \mathrm{~L}\right)$.

\begin{tabular}{|c|c|c|c|c|c|c|}
\hline \multirow{2}{*}{ № } & \multirow{2}{*}{ Modelo } & \multirow{2}{*}{$\mathrm{R}^{2}$} & \multicolumn{4}{|c|}{------------------Teste do modelo-----------------' } \\
\hline & & & $\mathrm{a}$ & $\mathrm{b}$ & $\mathrm{R}^{2}$ & RQME \\
\hline 1 & $\mathrm{AF}=1,7582 \mathrm{~L}^{1,7067}$ & 0,983 & 7,51 & 0,928 & 0,98 & 27,5 \\
\hline 2 & $\mathrm{AF}=0,5501 \mathrm{~L}^{2}+3,5589 \mathrm{~L}$ & 0,964 & 9,98 & 0,911 & 0,98 & 28,8 \\
\hline 3 & $A F=-0,000552 L^{3}+0,5712 L^{2}+3,3618 L$ & 0,959 & 9,70 & 0,912 & 0,98 & 28,8 \\
\hline 4 & $\mathrm{AF}=21,3668 \mathrm{~L}-118,9534$ & 0,941 & $-0,37$ & 0,912 & 0,95 & 45,9 \\
\hline 5 & $\mathrm{AF}=0,1328 \mathrm{C}^{2,5569}$ & 0,954 & $-46,78$ & 3,143 & 0,87 & 623,7 \\
\hline 6 & $\mathrm{AF}=0,9932 \mathrm{C}^{2}-5,3682 \mathrm{C}$ & 0,922 & 63,91 & 2,867 & 0,89 & 617,8 \\
\hline 7 & $A F=-0,041349 C^{3}+1,2039 C^{2}-7,2604 C$ & 0,923 & 177,34 & $-2,068$ & 0,75 & 787,0 \\
\hline 8 & $\mathrm{AF}=0,5961\left(\mathrm{C}^{*} \mathrm{~L}\right)^{1,0322}$ & 0,985 & $-4,94$ & 1,503 & 0,97 & 150,4 \\
\hline 9 & $\mathrm{AF}=0,7330(\mathrm{C} * \mathrm{~L})$ & 0,969 & 2,32 & 1,469 & 0,97 & 145,6 \\
\hline 10 & $\mathrm{AF}=0,000052\left(\mathrm{C}^{*} \mathrm{~L}\right)^{2}+0,6978\left(\mathrm{C}^{*} \mathrm{~L}\right)$ & 0,970 & $-9,47$ & 1,557 & 0,96 & 163,0 \\
\hline
\end{tabular}

Ciência Rural, v.39, n.5, ago, 2009. 


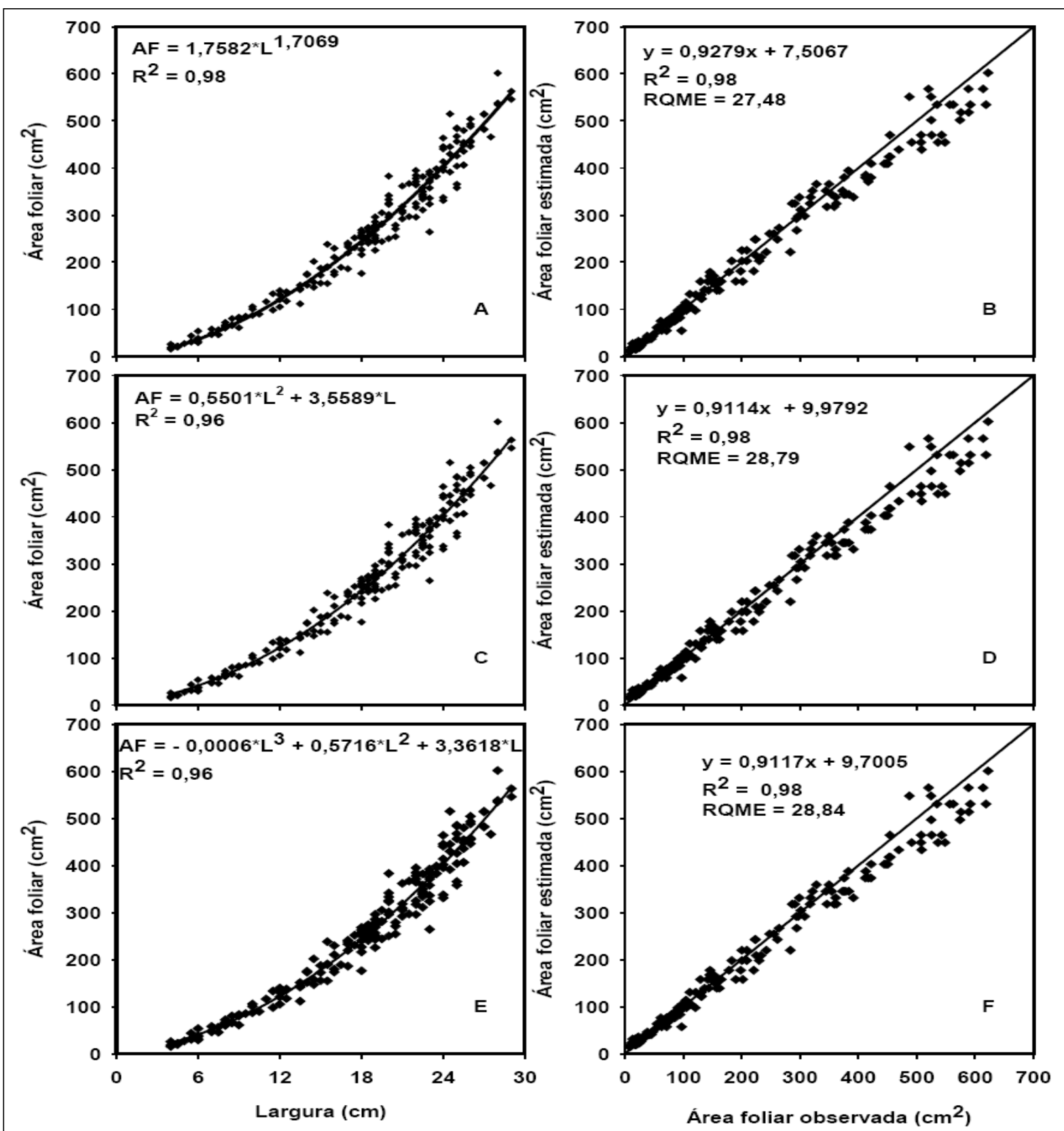

Figura 1 - Modelo de regressão não-linear potência (A), quadrático (C) e cúbico (E) e seus respectivos testes (B), (D) e (F), obtidos entre a largura da folha e a área do limbo foliar do girassol. Santa Maria, 2008.

Os modelos que utilizam somente a largura (L) ou o comprimento (C) são preferíveis, por utilizarem apenas uma das dimensões da folha. Com isso, há uma redução em 50\% no número de medições de dimensão linear a serem realizadas a campo. No caso do girassol, os resultados obtidos com os modelos demonstram que deve ser optada apenas a dimensão da largura, sendo o modelo exponencial capaz de se ajustar ao efeito da modificação de formato das folhas ao longo do ciclo de cultivo. Além disso, a medição da largura está sujeita a um erro experimental menor do que a do comprimento. Resultados nesse sentido foram encontrados para a cultura do morangueiro (PIRES et al., 1999) e da berinjela (COSTA et al., 2007).

Na figura 2, é apresentado o teste do modelo obtido por ROUPHAEL et al. (2007), onde se observa que esse modelo apresenta um baixo RQME, mas apresenta problemas para folhas pequenas, pois, para

Ciência Rural, v.39, n.5, ago, 2009. 


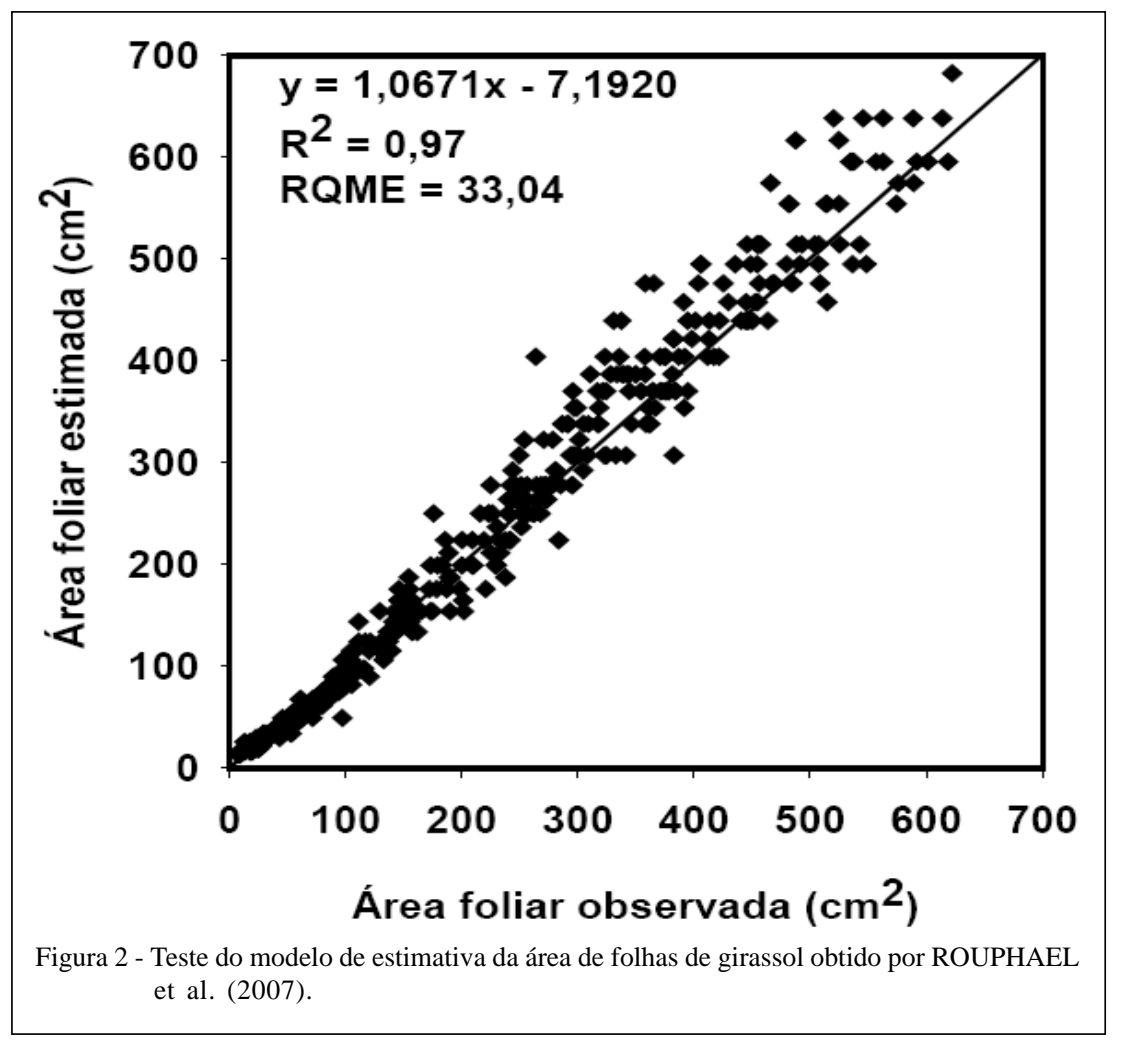

uma folha com $1 \mathrm{~cm}$ de largura, resultaria uma $\mathrm{AF}$ estimada de $7,37 \mathrm{~cm}^{2}$, o que não é realístico. A menor precisão do modelo proposto por ROUPHAEL et al. (2007) pode estar associada ao seu coeficiente linear, que, para folhas pequenas de girassol, superestima sua área foliar, provavelmente em função da diferença de formato entre folhas novas e completamente desenvolvidas. Folhas novas são proporcionalmente mais estreitas do que folhas completamente desenvolvidas. O girassol é uma cultura que apresenta uma rápida emissão de folhas e, portanto, a proporção de folhas pequenas é alta, o que pode resultar em um erro significativo. A mudança de formato da folha até seu completo desenvolvimento é a explicação para o fato de os modelos lineares não apresentam bom ajuste, mesmo quando incluem as duas dimensões (L e C) como variáveis independentes.

\section{CONCLUSÃO}

O modelo não-linear do tipo potência, utilizando a largura do limbo foliar, é o mais preciso e adequado para estimar a área das folhas de girassol, independentemente do seu tamanho.

\section{REFERÊNCIAS}

BLANCO, F.F.; FOLEGATTI, M.V. Padrão de distribuição de área foliar do pepino cultivado em ambiente protegido. Horticultura Brasileira, v.18, p.254-256, 2000. (Suplemento julho).

BLANCO, F.F.; FOLEGATTI, M.V. A new method for estimating the leaf area index of cucumber and tomato plants. Horticultura Brasileira, v.21, n.4, p.666-669, 2003. Disponível em: <http://www.scielo.br/scielo.php?pid=S010205362003000400019\&script=sci_arttext $>$. Doi: 10.1590/ S0102-05362003000400019.

BLANCO, F.F.; FOLEGATTI, M.V. Estimation of leaf area for greenhouse cucumber by linear measurements under salinity and grafting. Scientia Agrícola, v.62, n.4, p. 305-309, 2005. Disponível em: $<$ http://www.scielo.br/scielo.php?script=sci_arttext\&pid=S010390162005000400001>. Doi: 10.1590/S0103-90162005000400001

COELHO FILHO, M.A. et al. Estimativa da área foliar de plantas de lima ácida 'tahiti' usando métodos não-destrutivos. Revista Brasileira de Fruticultura, v.27, n.1, p.163-167, 2005. Disponível em: <http://www.scielo.br/scielo.php?pid=S010029452005000100043\&script=sci_arttext >. Doi: 10.1590/ S0100-29452005000100043.

COSTA, M. da. et al. Modelos de determinação não destrutiva da área foliar da berinjela cultivada em estufa plástica. In: CONGRESSO BRASILEIRO DE AGROMETEOROLOGIA, 15., 2007, Aracaju - SE. Anais... Aracaju: Sociedade Brasileira de Agrometeorologia, 2007. Disponível em CD-ROM. 
DE JESUS JR.,W.C. et al. Comparison of two methods for estimating leaf area index on common bean. Agronomy Journal v.93, p.989-991, 2001.

EMBRAPA. Sistema brasileiro de classificação de solos: Embrapa Produção de Informações. Rio de Janeiro : Embrapa Solos, 1999. 412p.

FAVARIN, J.L. et al. Equações para a estimativa do índice de área foliar do cafeeiro. Pesquisa Agropecuária Brasileira, v.37, n.6, p.769-773, 2002. Disponível em: <http:// w w w. s c i e l o.b r / s c i e lo.p h p ? p i d = S 0100 204X2002000600005\&script $=$ sci_arttext\&tlng=in $>$. Doi: 10.1590/S0100-204X2002000600005.

JANSSEN, P.H.M.; HEUBERGER, P.S.C. Calibration of process - oriented models. Ecological Modelling, v.83, n.1-2, p. 155-56, 1995. Disponível em: < http://www.sciencedirect.com/ science?_ob=ArticleURL\&_udi=B6VBS-4031 PNBD\&_user $=687358 \&$ \&doc $=1 \&$ \& $\mathrm{mt}=\&$ \&_orig $=$ search \&_sort $=$ d\&view $=$ c \&_acct $=$ C $000037899 \&$ \&_version $=1$ \&_urlversion $=0$ \&_us e ri d $=687358 \&$ m d $5=$ c877c89c9b72f82f45e7a328194b0eac>. Doi: 10.1016/03043800(95)00084-9.

LAKITAN, B. Empirical model for estimating leaf area in bean (Phaseolus vulgaris L.). Annual Report of the Bean Improvement Cooperative, v.32, p.19-21, 1989.

MORENO, J.A. Clima do Rio Grande do Sul. Porto Alegre: Secretaria da Agricultura, 1961. 46p.

NASCIMENTO, I.B. et al. Estimativa da área foliar do meloeiro. Horticultura Brasileira, v.20, n.4, p.555-558, 2002. Disponíve em: <http://www.scielo.br/scielo.php?script=sci_arttext\&pid=S010205362002000400009>. Doi: 10.1590/S0102-05362002000400009.

NIED, A.H. et al. Modelos para determinação não destrutiva da área das folhas do pepineiro cultivado em estufa plástica. Pesquisa Agropecuária Gaúcha, v.7, n.1, p.15-26, 2001.

PEKSEN, E. Non-destructive leaf area estimation model for faba bean (Vicia faba L.) Scientia Horticulturae, v.113, p.322328, 2007. Disponível em: < http://www.sciencedirect.com/ science?_ob=ArticleURL\&_udi=B6TC3-4NX8RKN$1 \&$ _user $=687358 \&$ \&doc $=1 \&$ \&mt $=\&$ \&_orig $=$ search $\&$ _sort $=$ d\&view $=$ c \&_acct $=$ C $000037899 \&$ \& versio $\mathrm{n}=1 \&$ _ u r l Ve r s i o $\mathrm{n}=0$ \&_ u s e r i d $=68735$ 8\&md5=1821dcb2ab2344603481ced2e2c5a4f9>. Di: 10.1016/ j.scienta.2007.04.003.

PEREIRA, A.R. et al. Evapo(transpi)ração. Piracicaba: FEALQ/ESALQ/USP, 1997. 70p.

PIRES, C. de M. et al. Estimativa da área foliar do morangueiro. Horticultura Brasileira, v.17, n.2, p.86-90, 1999

QUEIROGA, J.L. et al. Estimativa da área foliar do feijãovagem (Phaseolus vulgaris L.) por meio da largura máxima do folíolo central. Horticultura Brasileira, v.21, n.1, p.64-68, 2003. Disponível em: http://www.scielo.br/scielo.php?pid=S010205362003000100013\&script $=$ sci_arttext\&tlng $=$ pt. Doi: 10.1590/S0102-05362003000100013.

ROUPHAEL, Y. et al. Leaf area estimation of sunflower leaves from simple linear measurements. Photosynthetica, v.45, n.2, p.306-308, 2007. 